\title{
QUÍMICA ANALÍTICA NO BRASIL: ATUALIDADES, TENDÊNCIAS E DESAFIOS
}

\author{
Clésia Cristina Nascentes ${ }^{\mathrm{a}, *}$, Maria das Graças Andrade Korn ${ }^{\mathrm{b}}$ e Maria Valnice Boldrin Zanonic \\ aDepartamento de Química, Universidade Federal de Minas Gerais, 31270-901 Belo Horizonte - MG, Brasil \\ 'Instituto de Química, Universidade Federal da Bahia, 40170-115 Salvador - BA, Brasil \\ 'Instituto de Química, Universidade Estadual Paulista, 14800-060 Araraquara - SP, Brasil
}

Recebido em 04/04/2017; aceito em 11/05/20175

\begin{abstract}
CURRENT STATUS, TRENDS AND CHALLENGES ON ANALYTICAL CHEMISTRY IN BRAZIL. This work describes the development of Analytical Chemistry in Brazil from the Brazilian Chemical Society (SBQ) foundation to the present days. Important historical facts were highlighted and data on the human resources formation, financial investments and scientific production were used to evaluate and contextualize the progress achieved. The evolution of the scientific events of the area was also presented. The survey shows that Analytical Chemistry has advanced a lot in these 40 years, occupying a prominent position in the international scenario. This growth is mainly due to the formation of qualified human resources, the organization of scientific societies and inductive policies. It was also verified that the quantitative advance was greater than the qualitative one. Thus, one of the challenges for the next years is to work at the knowledge frontier and increases the quality and impact of scientific production in Analytical Chemistry. The evolution of the area into current trends such as Nanoscience, Microfluidic, Omics, Chemometric, Green Chemistry and Chemical Speciation is also presented and discussed.
\end{abstract}

Keywords: Analytical Chemistry; Brazil; Scientific production; Trends and challenges

\section{INTRODUÇÃO}

A Química brasileira tem apresentado um crescimento substancial nas últimas décadas e um dos alicerces é, sem dúvida, a Sociedade Brasileira de Química durante o período de 40 anos de atividades. A Química Analítica segue a mesma tendência de crescimento e alguns dados serão apresentados e discutidos.

Inicialmente, é importante destacar alguns marcos. Andrade e colaboradores ${ }^{1}$ apresentaram fatos históricos como o início da pesquisa científica em Química Analítica no Brasil em 1940 pelo Dr. Fritz Feigl e as importantes contribuições do Prof. Paschoal Senise em São Paulo, do Pe. Leopoldo Hainberger no Rio de Janeiro, do Prof. Antônio Celso Spinola Costa na Bahia e do Prof. Otto Alcides Ohlweiler no Rio Grande do Sul com a formação de químicos analíticos, que posteriormente foram nucleadores de grupos de pesquisa em diferentes universidades brasileiras.

Em 1977 foi criada a Sociedade Brasileira de Química (SBQ) e em julho de 1978 ocorreu a $1^{\text {a }}$ Reunião Anual da SBQ. Em julho de 1982, durante a $34^{\mathrm{a}} \mathrm{RASBPC} / 5^{\mathrm{a}} \mathrm{RASBQ}$, realizada em Campinas, SP, um grupo de pesquisadores reuniu-se no Instituto de Química da UNICAMP e decidiu criar um fórum para discussão das pesquisas desenvolvidas em Química Analítica no Brasil e, em novembro do mesmo ano, foi realizado o $1^{\circ}$ Encontro Nacional de Química Analítica na Pontifícia Universidade Católica do Rio de Janeiro. ${ }^{2}$ A Divisão de Química Analítica da SBQ foi criada em 1994, sendo a Profa. Carol Hollingworth Collins a primeira diretora dessa divisão.

Esse artigo busca descrever os avanços qualitativos e quantitativos na área de Química Analítica nestes 40 anos. Outros trabalhos já descritos na literatura trazem informações interessantes. Em 1985, o Prof. Paschoal Ernesto Américo Senise publicou um artigo no periódico Analytical Letters, reportando que havia trabalhos relevantes sendo desenvolvidos em Química Analítica no Brasil nessa época, mas que os grupos de pesquisa estavam centralizados em 10 universidades de alguns estados brasileiros (SP, RJ, MG, BA, PR, CE e DF). ${ }^{3}$ Oliveira

*e-mail: clesianascentes@ gmail.com
Neto et al. (2001) apresentaram os trabalhos desenvolvidos no período de 1995 a 2000 por pesquisadores e estudantes das principais universidades brasileiras com Programas de Pós-Graduação na área de Química Analítica. ${ }^{4}$

Em 2002, na edição especial da Química Nova dedicada aos 25 anos da SBQ, Fatibello Filho et al. descreveram o desenvolvimento da Química Analítica brasileira entre 1977 e 20025. Os autores avaliaram a literatura especializada e concluíram que os químicos analíticos do Brasil tinham uma significativa contribuição em periódicos científicos de alto impacto. Esses autores também fizeram uma interessante comparação entre os trabalhos apresentados no $11^{\circ}$ Encontro Nacional de Química Analítica (2001) e na XI European Conference on Analytical Chemistry (2000) e verificaram uma boa correlação entre as pesquisas realizadas no Brasil e no exterior na área de Química Analítica. Dentre as áreas consideradas como consolidadas destacavam-se espectrometria atômica e molecular, eletroanalítica, separações e análise em fluxo. Entre as áreas carentes, os autores citaram bioanalítica, miniaturização, especiação química, química analítica de processos e metrologia química. ${ }^{5}$

Em 2003, Zagatto e Sá discutiram o desenvolvimento da Química Analítica no Brasil ao longo do século XX em relação ao avanço científico internacional e as tendências para o século XXI. ${ }^{6}$ No que diz respeito às tendências, os autores destacaram a miniaturização de instrumentos, a redução no consumo de amostras e reagentes e menor produção de resíduos (química verde), a bioanalítica possibilitando análises in vivo e in situ e a necessidade de um maior controle na qualidade dos resultados gerados enfatizando a quimiometria, acreditação, rastreabilidade e exatidão. ${ }^{6}$ Verifica-se que as tendências são similares às áreas carentes que foram apontadas por Fatibello Filho e colaboradores ${ }^{5}$ em 2002. De Andrade et al. (2004) ${ }^{1}$ corroboraram as afirmações feitas por Zagatto e Sá ${ }^{5}$ e por Fatibello et al. ${ }^{6}$ sobre as áreas consolidadas, as tendências e carências da Química Analítica brasileira. Além disso, apresentaram uma importante reflexão sobre a necessidade de interação da academia com os setores industrial e de negócios, de forma que as pesquisas desenvolvidas na área de Química Analítica sejam mais direcionadas para novas tecnologias 
e inovação, contribuindo assim para o fortalecimento da indústria química no Brasil. ${ }^{1}$

Duas importantes revisões foram publicadas em 2008 e 2009 sobre o desenvolvimento da Química Analítica no Brasil. A primeira abordou os trabalhos publicados entre 2004 e 2008 em automação em química analítica, quimiometria, cromatografia, eletroforese capilar e espectrometria de massas, eletroanalítica, química analítica ambiental, procedimentos de pré-concentração e separação e espectroanalítica. ${ }^{7}$ A outra revisão abordou trabalhos publicados em 2008 e 2009 com enfoque nas aplicações dos métodos analíticos: meio ambiente, alimentos, produtos farmacêuticos, materiais biológicos, combustíveis e materiais. ${ }^{8}$

Assim, constata-se que os avanços da Química Analítica brasileira estão bem documentados e discutidos até 2009. Esses avanços podem ser atribuídos principalmente à formação de recursos humanos qualificados e ao incremento dos investimentos em educação, ciência e tecnologia. Além disso, os eventos científicos têm desempenhado um papel importante, pois promovem uma discussão entre pesquisadores e estudantes sobre vários aspectos da ciência e possibilitam a interação entre grupos de pesquisa do Brasil e do exterior, ampliando as colaborações e, consequentemente, a qualidade da produção científica. Dessa forma, serão descritos alguns aspectos importantes sobre esses dois tópicos.

A produção científica e tecnológica pode ser considerada um bom parâmetro, embora não seja o único, para se avaliar a qualidade e o impacto dos conhecimentos produzidos por uma determinada área e também a inserção da área no contexto internacional. Algumas informações foram levantadas sobre a produção brasileira em Química Analítica utilizando-se as bases de dados do ISI Web of Knowledge da Thomson Reuters. Recentemente, a Profa. Maria Valnice Boldrin Zanoni (UNESP) e o Prof. Sérgio Luís Costa Ferreira (UFBA) fizeram um excelente levantamento sobre a produção científica da Química Analítica no Brasil. Assim, dados desses dois levantamentos serão apresentados e discutidos neste artigo.

\section{FORMAÇÃO DE RECURSOS HUMANOS E INVESTIMENTOS FINANCEIROS}

A Pós-Graduação no Brasil existe desde 1930, no sistema de cátedra, mas os primeiros cursos de pós-graduação em Química no Brasil, no sistema formal, tiveram início na década de 60 do século XX. Em 1982 existiam somente seis Programas de Pós-Graduação (PPG) com área de concentração em Química Analítica aprovados pela $\mathrm{CAPES}^{2}$ e em 2000, o número de PPG era bem mais expressivo, sendo dezoito PPG's distribuídos em treze unidades da federação. A partir do ano 2000 o número de programas de PG em Química triplicou, principalmente devido à expansão do sistema universitário federal, via Programa de Apoio à Planos de Reestruturação e Expansão das Universidades Federais associado ao apoio fundamental da CAPES ao crescimento, consolidação e internacionalização do sistema de pós-graduação brasileiro. ${ }^{9}$

Atualmente, de acordo com o relatório do seminário de acompanhamento da CAPES, ${ }^{10}$ dos 66 PPG's em Química do Brasil, apenas dois não têm docentes que orientam na área de Química Analítica. Quatro estados da região norte (Acre, Amapá, Rondônia e Tocantins) ainda não possuem Programas de Pós-Graduação em Química. ${ }^{10}$ Percebe-se então uma descentralização e um aumento substancial na formação de recursos humanos em Química no Brasil (Figura 1). Os dados se referem a períodos que correspondem aos da avaliação da CAPES, que passou para quadrienal em 2013. Nos últimos 4 anos (2013 a 2016) foram titulados 2380 doutores em Química. Estima-se que aproximadamente um quarto do total de doutores titulados seja na área de Analítica, uma vez que na maioria dos programas ainda existe a divisão clássica nas 4 grandes áreas da Química (Analítica, Físico-química, Inorgânica e Orgânica).

Esse aumento impressionante na formação de recursos humanos que ocorreu em todas as áreas do conhecimento, só foi possível com o acréscimo dos investimentos. Nesse contexto é importante destacar o Programa de Apoio ao Desenvolvimento Científico e Tecnológico (PADCT), lançado em 1984 pelo governo federal. No total, o PADCT investiu US\$ 87,5 milhões no Subprograma de Química e Engenharia Química, sendo US\$34,9 milhões durante o PADCT-I (1985 a 1990) e mais US\$ 52,6 milhões durante o PADCT-II (1990 a 1995). ${ }^{13}$

O artigo de Paniago, publicado na Química Nova em 1997, descreveu os impactos positivos resultantes desses investimentos. ${ }^{14}$ Esses recursos foram utilizados principalmente na melhoria das bibliotecas, aquisição de equipamentos de grande porte e apoio aos cursos de graduação e pós-graduação em Química. No período de 1984 a 1995, esses investimentos resultaram em um aumento de 600\% na produção científica da área de Química em periódicos internacionais e o número de mestres e doutores titulados aumentou 200 e $300 \%$, respectivamente, representando uma grande contribuição para o desenvolvimento da área. ${ }^{13}$

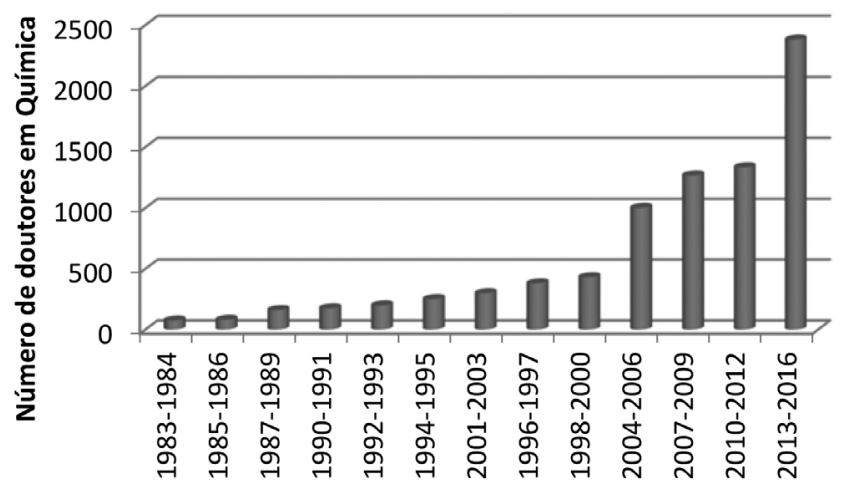

Figura 1. Número de doutores titulados em Química no Brasil no período de 1983 a 2016. FONTE: Relatórios CAPES ${ }^{9-12}$

No período de 2004 a 2015, os recursos investidos pela CAPES aumentaram 12 vezes, mas infelizmente observa-se uma redução nestes investimentos a partir de 2016. ${ }^{14}$ Outra importante iniciativa da CAPES foi a criação do Portal de Periódicos no final do ano 2000. O acesso facilitado e gratuito aos artigos científicos e outras bases de dados das mais importantes editoras do mundo, possibilita a atualização contínua de pesquisadores e estudantes de forma rápida e dinâmica.

Esse aumento na formação de recursos humanos em conjunto com a ampliação de vagas no ensino superior e o aumento dos investimentos dos governos estaduais, através das Agências de fomento estaduais (FAP's), e federais, principalmente por meio do CNPq, FINEP e CAPES, possibilitaram a nucleação e estruturação de grupos de pesquisa em quase todas as regiões do Brasil, com reflexos diretos sobre o aumento na produção científica e tecnológica da área de Química.

\section{OS EVENTOS CIENTÍFICOS}

Os eventos científicos são um importante fórum para discussão e troca de conhecimento entre pesquisadores, pós-graduandos, graduandos e profissionais de outros setores produtivos.

As Reuniões Anuais da Sociedade Brasileira de Química (RASBQ), que ocorrem desde 1978, e os Encontros Nacionais de Química Analítica, a partir de 1982, são dois importantes eventos que se consolidaram com o passar dos anos, nos quais pesquisadores e estudantes tem a oportunidade de expor e discutir os desenvolvimentos científicos na área de Analítica. 
O número de trabalhos de Química Analítica apresentados nas RASBQ, entre 1978 e 2016, pode ser visualizado na Figura 2. Geralmente a Química Analítica figura entre as 5 áreas com maior número de trabalhos nas RASBQ, o que demonstra a maturidade e importância da área no contexto nacional. De uma forma geral, até 2011 houve uma tendência de crescimento, com algumas flutuações. No Ano Internacional da Química (2011) foi realizada a maior RASBQ e foram apresentados 383 trabalhos de Analítica. A partir de 2012 observa-se uma redução no número de trabalhos apresentados, o que se deve em parte à mudança no número de trabalhos que poderia ser submetido por inscrição (dois trabalhos/inscrição até 2011 e um trabalho/inscrição a partir de 2012). Outro fator que talvez tenha contribuído, foi o aumento do número de eventos científicos específicos, como será descrito posteriormente.

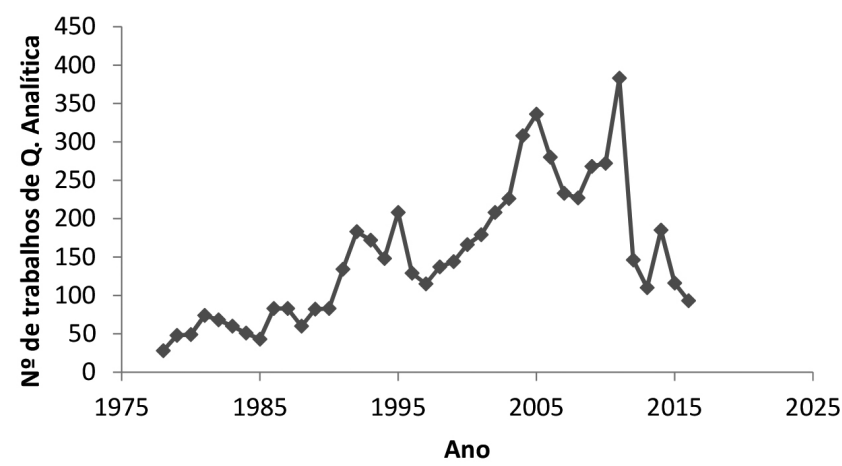

Figura 2. Número de trabalhos de Química Analítica apresentados nas Reuniões Anuais da Sociedade Brasileira de Química no período entre 1978 e 2016

Do $1^{\circ}$ ao $18^{\circ}$ ENQA, o número de trabalhos apresentados foi quase sempre crescente, passando de 136 para 1011, respectivamente. Hoje, o ENQA é considerado o evento de Química Analítica mais importante da América Latina. As três últimas edições contaram com mais de 1000 participantes do Brasil e também de outros países. Um panorama geral da Química Analítica brasileira pode ser obtido observando-se a distribuição de trabalhos apresentados no ENQA, uma vez que é possível avaliar as subáreas que mais se destacam no cenário nacional e também linhas de pesquisa em ascensão. A Química Analítica está em constante transformação em função de novas demandas e dos desenvolvimentos tecnológicos que abrem novas possibilidades de pesquisa. Para avaliar essa evolução, é comparada a distribuição de trabalhos por temas no $8^{\circ}$ ENQA (1995) e no $18^{\circ}$ ENQA (2016) (Figura 3). Em 1995, 75\% dos trabalhos apresentados no $8^{\circ}$ ENQA eram das subáreas de espectroanalítica, eletroanalítica, química ambiental e técnicas de separação. O número de trabalhos envolvendo automação, instrumentação e informática era pequeno (8\%). O restante dos trabalhos se dividia nas áreas de análise térmica, equilíbrio químico, educação em química e radioanalítica.

Após 21 anos verifica-se uma maior distribuição dos trabalhos entre as subáreas. A Química Ambiental manteve a mesma proporção de trabalhos, mas a espectroanalítica apresentou uma redução de aproximadamente $50 \%$, eventualmente porque parte desses trabalhos passou a ser apresentado em subáreas mais recentes, tal como preparo de amostras. As subáreas de tratamento de amostras e quimiometria, que não estavam claramente definidas no $8^{\circ}$ ENQA, tiveram uma contribuição importante no $18^{\circ}$ ENQA com $14 \%$ e $10 \%$ dos trabalhos, respectivamente. As áreas de eletroanalítica e técnicas de separação tiveram uma pequena redução nesse período, provavelmente em função da consolidação de congressos específicos dessas subáreas. Um número de trabalhos significativo (5\%) na área de Química Analítica e Energia demonstra que o Brasil tem massa crítica atuando nesse tema, o que também reflete indução de agências
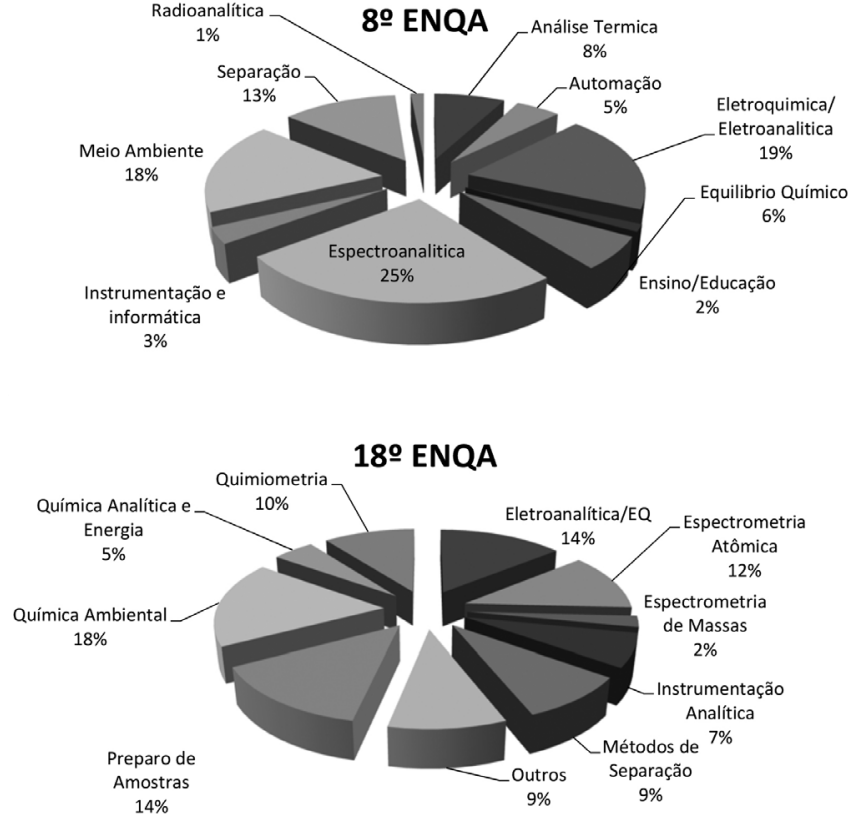

Figura 3. Distribuição dos trabalhos por temas no $8^{\circ}$ (1995) e $18^{\circ}$ (2016) Encontros Nacionais de Química Analítica

de fomento. Vale destacar que $9 \%$ dos trabalhos não se encaixaram nos temas previstos pela Comissão Organizadora, o que indica que novas linhas de pesquisa têm surgido no Brasil, contribuindo para uma maior diversidade científica.

É importante também destacar a criação do Congresso Iberoamericano de Química Analítica (CIAQA) que foi proposto com a intenção de estruturar e fortalecer as interações e cooperações entre as comunidades de Química Analítica da América Latina e dos países da Península Ibérica. O $1^{\circ}$ CIAQA teve suas atividades alicerçadas pelo $13^{\circ}$ ENQA, o qual ocorreu em 2005, na cidade de Niterói (RJ). A adesão de pesquisadores de Química Analítica provenientes de outros países foi relativamente discreta nessa primeira edição do CIAQA (50 participantes vindos da Argentina, Chile, Uruguai, México, Venezuela, Portugal e Espanha) para um total de 750 inscritos no ENQA. Nesse evento, os Grupos de Pesquisa em Química Analítica presentes apresentaram seus interesses, trabalhos e infraestrutura de pesquisa e discutiram a criação da Rede Ibero-Americana de pesquisa em Química Analítica, além de participarem das atividades usuais do ENQA. Os eventos posteriores foram realizados em diferentes países da América Latina (Argentina, Brasil, Chile, Uruguai e México) e o $7^{\circ}$ CIAQA será realizado novamente no Brasil em conjunto com o $19^{\circ}$ Encontro Nacional de Química Analítica em outubro de 2018. O CIAQA vem cumprindo o papel de aumentar as interações entre pesquisadores latino-americanos.

Além da RASBQ e ENQA, muitos eventos científicos, normalmente mais específicos, contam com grande participação de químicos analíticos brasileiros. O Simpósio Brasileiro de Eletroquímica e Eletroanalítica (SIBEE) ocorre bianualmente desde 1978 e teve sua $21^{\text {a }}$ edição em 2017 . No $20^{\circ}$ SIBEE, dos 570 trabalhos apresentados, 162 eram de eletroanalítica e 86 de sensores e biossensores, perfazendo um total de 248 trabalhos (43,5\%). Na área de espectrometria atômica, desde 1988 ocorre bianualmente o Rio Symposium on Atomic Spectrometry. Esse evento foi criado no Brasil, pelos Profs. Adilson José Curtius e Bernhard Welz com o intuito de congregar pesquisadores e estudantes da América Latina que atuam na área de espectrometria atômica. Esse evento já teve 7 edições realizadas no Brasil e as outras edições foram realizadas na Argentina (2), Chile (1), Venezuela (2) e México (2), sempre com uma 
significativa participação de brasileiros. Além de latino-americanos, o Rio Symposium recebe renomados pesquisadores de outros países, tais como Estados Unidos, Canadá, Itália, Áustria, Escócia, Espanha e Portugal, sendo responsável por promover novas colaborações científicas entre brasileiros e pesquisadores de outros países.

$\mathrm{Na}$ área de cromatografia, dois importantes eventos devem ser destacados: o Congresso Latino Americano de Cromatografia (COLACRO), que teve início no Brasil em 1986 e é realizado a cada 2 anos em diferentes países da América Latina, e o Simpósio Brasileiro de Cromatografia e Técnicas Afins (SIMCRO), realizado pela primeira vez em 1996 e bianualmente a partir de 2006, após uma reformulação. Esses eventos contam sempre com uma participação massiva e um grande número de trabalhos apresentados: no XVI COLACRO (realizado pela primeira vez fora da América Latina - Portugal) foram 198 trabalhos apresentados e no SIMCRO 2014, 279 trabalhos.

O Encontro Nacional de Química Ambiental (ENQamb), criado em 1990, é realizado bianualmente desde sua $3^{\text {a }}$ edição (2006). Em sua $7^{\mathrm{a}}$ edição (2014), foram apresentados 138 trabalhos científicos.

Em 2004, De Andrade e coautores discutem sobre a relevância estratégica da interação do meio acadêmico com o setor industrial/ empresarial. Existe ainda um grande descompasso entre a ciência e tecnologia produzidas nos laboratórios de universidades e centros de pesquisa e o que é praticado no cotidiano de outros setores produtivos. ${ }^{1}$ Com o intuito de facilitar essa interação, numa importante iniciativa, foi criado em 2009 o Congresso Analítica Latin America que acontece conjuntamente com a Feira Analítica Latin America. A Feira Analítica se posiciona como plataforma geradora de negócios com a presença de marcas multisetoriais, que oferecem soluções de qualidade para o mercado analítico. O Congresso Analítica caminha para sua $5^{\circ}$ edição e conta com um número crescente de trabalhos apresentados (141 trabalhos na $4^{\mathrm{a}}$ edição). A programação sempre aborda temas atuais e discussões enriquecedoras entre os setores acadêmico e empresarial.

Nos últimos anos, eventos mais específicos como o Encontro Brasileiro sobre Especiação Química (ESPEQBrasil - na $5^{\text {a }}$ edição), a Conferência da Sociedade Brasileira de Espectrometria de Massas (BrMass - na $6^{\text {a }}$ edição), o Workshop de Quimiometria (na $8^{\text {a edição) }}$ e o Workshop em Microfluídica (na $7^{\mathrm{a}}$ edição) têm se consolidado, sempre com número crescente de participantes e trabalhos apresentados. Isso demonstra o importante desenvolvimento dessas linhas de pesquisa no Brasil, acompanhando as tendências mundiais.

\section{PRODUÇÃO CIENTÍFICA}

A avaliação da produção cientifica dos químicos analíticos brasileiros, em termos quantitativos e qualitativos, foi um dos critérios adotados para mensurar os avanços e internacionalização da área.

Para essa avaliação, realizou-se um levantamento no período de 1977 a 2016 e os dados foram organizados por quinquênios. Inicialmente selecionaram-se 12 periódicos da área, que apresentam elevado fator de impacto: Analytical Chemistry (FI= 5,886), Microchimica Acta $(\mathrm{FI}=4,831)$, Sensors and Actuators B Chemical $(\mathrm{FI}=4,758)$, Analytica Chimica Acta $(\mathrm{FI}=4,712)$ Talanta $(\mathrm{FI}=$ $4,035)$, Analyst (FI = 4,033), Journal of Chromatography A (FI = 3,926), Journal of Analytical Atomic Spectrometry ( $\mathrm{FI}=3,379$ ), Spectrochimica Acta B Atomic Spectroscopy $(\mathrm{FI}=3,289)$, Analytical and Bioanalytical Chemistry ( $\mathrm{FI}=3,125)$, Microchemical Journal $(\mathrm{FI}=2,893)$ e Journal of Electroanalytical Chemistry $(\mathrm{FI}=2,822)$. Oito desses periódicos publicam trabalhos de todas as subáreas da Química Analítica e os outros quatro, apesar de serem mais específicos, englobam algumas das áreas mais consolidadas no Brasil: técnicas de separação, espectroanalítica, eletroanalítica, sensores e microssistemas. Vale destacar que a Química Analítica é uma área com grande interação com as outras áreas da Química e também com outras áreas do conhecimento, tais como biologia, medicina, farmácia, física, engenharias e agronomia. Dessa forma, os químicos analíticos atuam em diferentes áreas e publicam também em periódicos da área de alimentos, meio ambiente, energia, medicamentos, análises clínicas, ciências forenses, materiais, dentre outras.

A Figura 4 mostra o número de publicações de pesquisadores brasileiros nos 12 periódicos selecionados no período de 1977 a 2016. De 1977 a 1996 houve um aumento de aproximadamente $358 \%$ e se considerarmos o período todo, o aumento foi de $2.373 \%$ ou seja, a produção dos químicos analíticos brasileiros nesses importantes periódicos aumentou certa de 24 vezes no período de 40 anos. Esse aumento acompanhou o crescimento no número de doutores titulados em Química no Brasil, que de 1982 para 2016 aumentou 16 vezes.

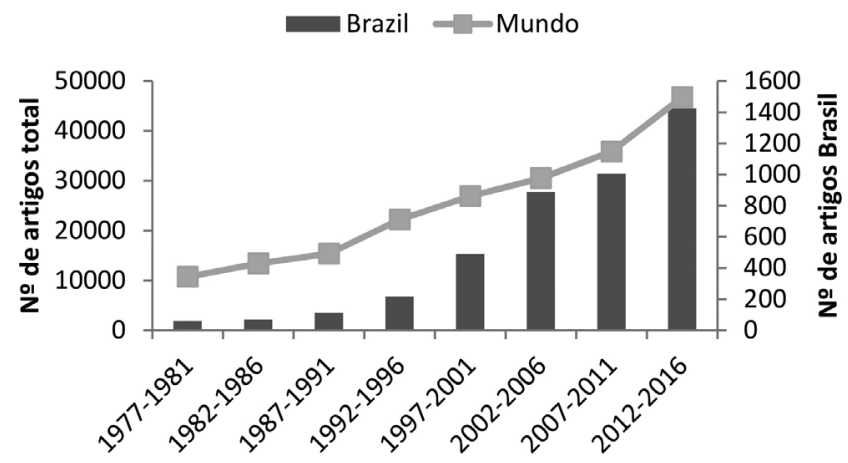

Figura 4. Número de artigos total e de artigos publicados por brasileiros no período de 1977 a 2016 em doze importantes revistas de Química Analítica, segundo levantamento no ISI Web of Knowledge da Thomson Reuters

Esse aumento no número de doutores titulados e na produção científica da área resultou em um aumento também na demanda de bolsas de produtividade do CNPq. Em 2009, a área de Química tinha 604 bolsistas de produtividade, sendo 23,5\% da Química Analítica. ${ }^{15}$ De acordo com dados do CNPq, em 2016 o número de bolsistas de produtividade passou para 707 (17\% de aumento), dos quais $22,8 \%$ pertencem a Química Analítica. A distribuição destes pesquisadores mostra que a grande maioria (56,2\%) são bolsistas nível 2, 20,5\% são bolsistas nível 1D, 7,45\% são bolsistas nível 1C, 6,83\% nível 1B, $8,07 \%$ nível $1 \mathrm{~A}$, e apenas um bolsista $(0,62 \%)$ na modalidade sênior. A grande maioria destes bolsistas está na região Sudeste $(54,7 \%)$, seguido de $24,2 \%$ na região Sul, $19,3 \%$ no Nordeste, $1,24 \%$ no Centro-oeste e apenas $0,62 \%$ no Norte.

Esse perfil de distribuição regional dos bolsistas é o mesmo observado para a produção científica sendo que no período de 2006 a 2015 , a região sudeste respondeu por $60 \%$ da produção brasileira na área, seguida das regiões Sul $(18,8 \%)$ e Nordeste $(16,5 \%)$. Nesse período houve um aumento significativo na produção científica do Nordeste e também do Centro-Oeste e Norte, provavelmente devido às políticas indutivas do $\mathrm{CNPq}$ e à nucleação de novos grupos de pesquisa nessas regiões. Destaca-se que 22 (52\%) dos Programas de Pós-graduação com mestrado e doutorado estão no Sudeste, sendo 9 deles no estado de São Paulo que foi responsável por $74 \%$ da produção científica do Sudeste entre 2006 e 2015. Além disso, o Sudeste detém a maior parte dos Programas de Pós-Graduação de excelência em Química (classificados pela CAPES com notas 6 e 7): 8 na região Sudeste, 4 na região Sul e apenas 1 no Nordeste. Esses dados evidenciam a importância da Pós-Graduação na produção de conhecimento científico no Brasil.

Para se avaliar melhor o crescimento quantitativo e qualitativo da área, outras informações referentes aos artigos publicados nas 12 revistas escolhidas estão compiladas na Tabela 1. 
Tabela 1. Informações sobre a produção científica da Química Analítica brasileira em 12 revistas representativas da área, segundo levantamento no ISI Web of Knowledge da Thomson Reuters

\begin{tabular}{lccccc}
\hline Período & $\begin{array}{c}\mathrm{N}^{\mathrm{o}} \text { de } \\
\text { artigos }\end{array}$ & $\begin{array}{c}\text { \% artigos } \\
\text { brasileiros }\end{array}$ & $\begin{array}{c}\text { Colocação }^{\mathrm{b}} \\
\text { no ranking }^{2}\end{array}$ & Citações $^{\mathrm{c}}$ & $\begin{array}{c}\text { \% autocita- } \\
\text { ções }\end{array}$ \\
\hline $1977-1981$ & 60 & 0,56 & 29 & 1922 & 4,42 \\
$1982-1986$ & 68 & 0,51 & 32 & 1484 & 2,36 \\
$1987-1991$ & 111 & 0,72 & 29 & 2010 & 1,99 \\
$1992-1996$ & 215 & 0,97 & 20 & 5745 & 1,04 \\
$1997-2001$ & 489 & 1,82 & 13 & 17024 & 2,23 \\
$2002-2006$ & 888 & 2,91 & 10 & 27466 & 3,76 \\
$2007-2011$ & 1005 & 2,80 & 11 & 27712 & 3,97 \\
$2012-2017$ & 1424 & 3,05 & 12 & 10077 & 12,7 \\
\hline
\end{tabular}

${ }^{a}$ Porcentagem calculada considerando-se o número total de artigos publica-

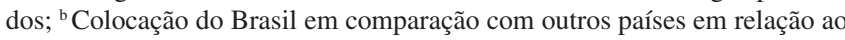
número de artigos publicados; 'Somatório das citações dos artigos publicados no período descrito, de acordo com o ISI Web of Knowledge no dia 27 de fevereiro de 2017.

No período avaliado a porcentagem de artigos com autores brasileiros nestes 12 periódicos quintuplicou, passando de $0,56 \%$ no período de 1977 a 1982 para 3,05\% no último quinquênio. O mesmo panorama foi observado no levantamento realizado pelos professores Maria Valnice e Sérgio Ferreira em 92 periódicos da área de Química Analítica: no período de 1982 a 1986 o Brasil contribuía com 0,36\% da produção mundial em Química Analítica e no período de 2011 a 2015 a contribuição brasileira foi de $2,96 \%$. Nesse levantamento mais amplo, verificou-se que o número de artigos brasileiros com pelo menos 1 co-autor de outro país aumentou $731 \%$ no período de 1994 a 2014, o que reflete a desejável intensificação das colaborações internacionais.

A posição do Brasil no ranking do número de trabalhos publicados melhorou, sendo que até 1991 ocupávamos o $29^{\circ}$ lugar e no período de 2002 a 2016 , passamos para posições variando de $10^{\circ}$ ao $12^{\circ}$ (Tabela 1). Atualmente, os primeiros lugares desse ranking são: China $\left(1^{\circ}\right)$, Estados Unidos $\left(2^{\circ}\right)$ e Espanha $\left(3^{\circ}\right)$. No quinquênio anterior (20072011) os EUA ocupavam o $1^{\circ}$ lugar e a China o $2^{\circ}$. O crescimento da China nos últimos anos foi tão acentuado que ultrapassou os EUA. Comparando-se o período de 2007 a 2011 com o período de 2012 a 2016, a China praticamente dobrou o número de publicações nesses periódicos. Mesmo considerando que a população da China é cerca de 4 vezes maior do que a população dos EUA, esse rápido crescimento na produção científica impressiona.

O número total de citações dos trabalhos de pesquisadores brasileiros tem aumentado quase que na mesma proporção do aumento do número de trabalhos, indicando que o impacto da produção brasileira também tem aumentado. Considerando o número de citações dos trabalhos publicados no período de 2002 a 2016, o Brasil ocupa a $11^{\text {a }}$ colocação e os primeiros colocados são os EUA $\left(1^{\circ}\right)$, China $\left(2^{\circ}\right)$ e Espanha $\left(3^{\circ}\right)$. Como pode ser observado, de forma geral existe uma boa concordância nos rankings do número de trabalhos publicados e do número total de citações desses trabalhos. Em termos de autocitações, o Brasil apresenta um percentual baixo, sendo menor que $5 \%$ na maior parte do período avaliado. No último quinquênio esse percentual subiu significativamente, passando de $3,97 \%$ para $12,7 \%$. Esse mesmo panorama foi observado também para os outros países, indicando que aparentemente o número de autocitações é maior nos anos iniciais pós-publicação. De acordo com Fatibello Filho et al. (2002) é importante um equilíbrio entre citações locais, que denotam a produção prévia e o processo de consolidação da área, e citações internacionais, que mostram o entrelaçamento com a química analítica mundial. ${ }^{6}$

Outra forma de se avaliar a qualidade da produção científica é através do número de citações por artigo. Esse critério minimiza algumas distorções que podem ser causadas por diferenças em relação ao tamanho e população dos países avaliados. Embora seja difícil determinar com precisão o aumento no número de citações/artigo, estima-se que que houve um aumento em torno de $50 \%$, quando se comparam as citações dos trabalhos publicados entre 1982 e 1986 e aqueles publicados entre 2002 e 2006. Artigos mais recentes não foram considerados, pois poderiam prejudicar essa comparação, já que o número de citações aumenta com o tempo posterior à publicação. O aumento no número de citações/artigo não acompanhou o aumento no número de artigos publicados. Isso poderia indicar que avançamos mais rapidamente em aspectos quantitativos do que qualitativos ou sob outra perspectiva, mais otimista, poderíamos concluir que mesmo tendo aumentado expressivamente o número de publicações, conseguimos manter e até melhorar gradualmente a qualidade do que é produzido. Contudo, é notório que o Brasil passa a ser um dos protagonistas importantes no cenário mundial e é provável que a consolidação da tradição científica trará uma expansão da visibilidade. Avaliar a qualidade da produção científica não é uma tarefa fácil e os critérios utilizados podem ser questionados. De qualquer forma, é interessante observar o que acontece com o ranking quando se considera o número de citações por artigo (no período de 2002-2016 para os 12 periódicos selecionados na área de Química Analítica): Singapura $\left(1^{\circ}\right)$, EUA $\left(2^{\circ}\right)$, Holanda $\left(3^{\circ}\right)$, Inglaterra $\left(4^{\circ}\right)$ e Suécia $\left(5^{\circ}\right)$. A China ocupa o $27^{\circ}$ e o Brasil o $31^{\circ}$. Apesar da pequena dimensão, Singapura apresenta o maior Índice de Desenvolvimento Humano (IDH) dos países asiáticos ( $9^{\circ}$ melhor do mundo em 2014) e tem um dos maiores PIB per capita do mundo (US\$ 53,224.00). ${ }^{16}$ Os cinco países com maior número de citações/artigo têm IDH $(0,91$ a 0,92 ) e PIB per capita (US\$ 49,000.00 a US\$ 56,000.00) próximos. Nesses índices, Brasil e China são semelhantes: Brasil com IDH e PIB per capta de 0,71 e US\$ 8,802.00, respectivamente, e China com IDH de 0,73 e PIB per capita de US\$ 8,290.00. ${ }^{16}$

O desenvolvimento científico e tecnológico de qualquer país depende de continuidade de investimentos e políticas bem definidas de ciência, tecnologia e inovação. Como já discutido, nos últimos 25 anos os Governos Federal e Estadual realizaram investimentos significativos que colocaram o Brasil em destaque em termos de produção científica e tecnológica. Já avançamos muito, mas os investimentos devem ser contínuos para que possamos ter e manter uma infraestrutura adequada para trabalharmos nas fronteiras do conhecimento, buscando um crescimento quantitativo e qualitativo que nos permita dar respostas e soluções adequadas para os diversos problemas da sociedade moderna.

\section{TENDÊNCIAS E DESAFIOS}

Nos últimos 40 anos, a ciência no Brasil tem avançado a passos largos e a Química Analítica acompanhou esse avanço, como pode ser verificado pelos dados apresentados. É inegável que alcançamos um novo patamar e ocupamos um lugar de destaque no cenário internacional. A formação de recursos humanos, os investimentos em infraestrutura, a organização das sociedades científicas e as políticas indutoras estabelecidas principalmente pela CAPES e pelo CNPq tiveram papel fundamental nessa evolução, assim como a consolidação de agências de fomento estaduais.

Os dados também possibilitam inferir que avançamos mais em termos quantitativos que qualitativos. Assim, nosso maior desafio, como produtores de ciência, tecnologia e inovação no Brasil, é elevar a qualidade e o impacto da produção científica e tecnológica 
do país. Para isso é importante trabalhar nas fronteiras do conhecimento, acompanhando tendências e buscando inovações. A Química Analítica é uma área que permeia e interage com várias outras áreas do conhecimento e isso aumenta as possibilidades de crescimento e também os desafios.

Em 2014, a FAPESP publicou em sua revista mensal o texto "Ciência em Evolução", reportando um estudo realizado por dois pesquisadores da University London College indicando mudanças na produção científica mundial. ${ }^{17}$ De acordo com um dos autores, "Novas áreas científicas trazem com elas novas oportunidades de crescimento e conexões com outros campos já estabelecidos". Ele comenta ainda sobre o dilema entre apoiar excelência científica em áreas consolidadas e, ao mesmo tempo, seguir novas tendências e garantir a relevância dos seus sistemas de ciência em áreas emergentes. Esse é um desafio pertinente à todas as áreas do conhecimento e caberia uma discussão/reflexão mais aprofundada. A Sociedade Brasileira de Química poderia liderar essa discussão, que seria certamente muito benéfica para nossa comunidade. Esses autores afirmam também que regiões como América Latina e Ásia, que apresentam índices de publicação altos, mas ainda com impacto relativo mais baixo, caracterizam-se pela maior capacidade de absorver conhecimento utilizando modelos desenvolvidos em outros países. Já as regiões com índices de citação mais elevados são aquelas que estão na fronteira do conhecimento. ${ }^{17}$ Essa afirmação corrobora os dados anteriormente apresentados. A Química Analítica brasileira dispõe de material humano altamente qualificado, tem produzido uma grande quantidade de artigos com um número significativo de citações e, tal como a maioria das áreas em ciências no Brasil, agora precisa avançar na qualidade e no impacto da sua produção científica.

Em 2002 e 2003 foram publicados dois artigos que mostravam os avanços e as tendências da Química Analítica, enfatizando áreas que deveriam ser priorizadas como: bioanalítica, miniaturização, especiação química, química analítica de processos, metrologia química, química verde, quimiometria. ${ }^{5,6}$ Buscou-se então avaliar o avanço do Brasil nestes temas. Para isso, selecionaram-se algumas palavras-chave e realizou-se uma nova pesquisa na base de dados ISI Web of Knowledge, utilizando-se como filtros a área de Química Analítica e o país, Brasil. O período selecionado para essa pesquisa foi de 2002 a 2016. Compilou-se o número de trabalhos de autores brasileiros publicados nos temas escolhidos e a colocação do Brasil em cada uma das áreas em comparação com os outros países do mundo (Tabela 2). Em todos os temas, observou-se um aumento crescente de trabalhos publicados no período avaliado. Algumas áreas como microfluídica, nanociências e "Omics" não haviam sido diretamente mencionadas nos trabalhos publicados há 15 anos. São temas atuais que ainda podem ser muito explorados. A microfluídica tem possibilitado a miniaturização de diversos sistemas analíticos, facilitando a portabilidade e as análises in situ. As "Omics" têm ampliado muito o conhecimento sobre os sistemas biológicos, auxiliando, entre outras coisas, na compreensão dos mecanismos relacionados à determinadas doenças e também na busca por novos tratamentos. A Nanociência tem ampliado as possibilidades para sistemas analíticos, mas traz novos desafios como a necessidade de métodos adequados para a determinação de impurezas e o controle da produção de nanomateriais, estudos na área de nanotoxicologia, avaliação dos impactos ambientais resultantes da disposição destes materiais etc.

De uma forma geral, verifica-se que a Química Analítica do Brasil se destaca nas áreas de metrologia química, quimiometria, química verde, técnicas de microextração e técnicas de análise direta. O número de trabalhos encontrados em Química Analítica para áreas interdisciplinares como por exemplo Química Verde, "Omics" e Nanociências pode ter sido subestimado, pois muitos químicos analíticos preferem publicar em revistas especializadas. $\mathrm{O}$ número de trabalhos publicados em nanociência, microfluídica e especiação química é significativo e o Brasil está bem colocado ( $11^{\circ}$ lugar). De acordo com esse levantamento, as áreas de bioanálise e "Omics" ainda são incipientes no Brasil, com um pequeno número de publicações e as piores colocações em comparação com outros países.

Tabela 2. Número de trabalhos publicados por pesquisadores brasileiros e colocação do Brasil em comparação com outros países do mundo em temas atuais (ISI Web of Knowledge)

\begin{tabular}{lcc}
\hline Palavras-chave & $\begin{array}{c}\mathrm{N}^{\mathrm{o}} \text { de } \\
\text { trabalhos }\end{array}$ & $\begin{array}{c}\text { Colocação Brasil no } \\
\text { Ranking Mundial }\end{array}$ \\
\hline Accreditation, Traceability, Validation & 896 & $7^{\circ}$ \\
$\begin{array}{l}\text { Chemometric, multivariate } \\
\text { optimization, multivariate calibration }\end{array}$ & 403 & $4^{\circ}$ \\
Microextraction & 369 & $6^{\circ}$ \\
Nanomaterials, Nanotubes, & 259 & $11^{\circ}$ \\
Nanosystems & & \\
Speciation & 229 & $11^{\circ}$ \\
Microfluidic & 113 & $11^{\circ}$ \\
Direct analysis, solid analysis & 82 & $5^{\circ}$ \\
Metabolomics, metalomics, proteomics & 66 & $21^{\circ}$ \\
Green Chemistry, Green Method, & 52 & $2^{\circ}$ \\
Green Procedure & & $16^{\circ}$ \\
Bioanalysis, in vivo analysis & 33 &
\end{tabular}

Curiosamente, as áreas de maior destaque são as que requerem menor investimento em termos de infraestrutura. Apesar dos recursos destinados à ciência e tecnologia nos últimos anos, é comum encontrar na maior parte das universidades brasileiras uma grande defasagem em termos de equipamentos de última geração, principalmente equipamentos de grande porte. Somam-se a isso as crises política e econômica persistentes que assolam nosso país. Os cortes que vêm sendo realizados pelo Governo Federal na área de Ciência e Tecnologia e a situação precária de muitas FAP's colocam em risco os avanços duramente alcançados. Sem investimentos, a ciência brasileira poderá estagnar ou até mesmo retroceder. Avançar na fronteira do conhecimento requer continuidade de investimentos e visão dos governantes. É preciso absoluta clareza que ciência, tecnologia e inovação estão diretamente relacionadas com a qualidade de vida da população e também com o desenvolvimento econômico do país. Em épocas de severos cortes orçamentários, é fundamental ter a sapiência para entender quais cortes acabam por inviabilizar um futuro promissor e soberano.

\section{AGRADECIMENTOS}

Os autores agradecem a Alessandro Santos Borges e Sérgio Luís da Costa Ferreira pelo auxílio em levantamentos bibliográficos, a Joaquim de Araújo Nóbrega pela revisão do texto e a Diretoria da SBQ pelo convite para elaboração deste artigo.

\section{REFERÊNCIAS}

1. de Andrade, J. B.; Korn, M. G.; Cadore, S.; Microchem. J. 2004, 77, 101.

2. Collins, C. H.; Cadore, S.; J. Braz. Chem. Soc. 2003, 14, 1.

3. Senise, P. E.A.; Anal. Lett. 1985, 18, 1933.

4. de Oliveira Neto, G.; Cadore, S.; Kubota, L. T.; Anal. Lett. 2001, 34, 471.

5. Fatibello-Filho, O.; Neves, E. A.; Rocha, F. R. P.; Nobrega, J. A.; Quim. Nova 2002 25, 62. 
6. Zagatto E. A. G.; Sá S. M. O.; J. Braz. Chem. Soc. 2003, 14, 153.

7 Ferreira, S. L. C.; Pereira, P. A. P.; Nóbrega, J. A.; Fatibello-Filho, O.; Feres, M. A.; Reis, B. F.; Bruns, R. E.; Aquino Neto, F. R.; Anal. Lett. 2008, 41, 1494.

8. Rocha, F. R. P.; Pereira-Filho, E. R.; Nóbrega J. A.; J. Braz. Chem. Soc. 2009, 20, 1759.

9. Monteiro, A. L.; Furlan, M.; Suarez, P. A. Z.; Documento de Área 2017, CAPES. http://www.capes.gov.br/images/documentos/Documentos_de_ area_2017/04_QUIM_docarea_2016.pdf. Acessado em 15 de março de 2017.

10. Monteiro, A. L.; Furlan, M.; Suarez, P. A. Z.; Relatório do Seminário de Acompanhamento 2015, CAPES. http://www.capes.gov.br/images/ documentos/Relatorios_seminario_acompanhamento_2015/04_QUIM_ RelSem_2015.pdf Acessado em 02 de março de 2017

11. Dias, L. C.; Monteiro, A. L., Nóbrega, J. A.; Documento de área 2013, CAPES. http://www.capes.gov.br/images/stories/ download/avaliacaotrienal/Docs_de_area/Quimica_doc_area_e_ comiss\%C3\%A3o_att08deoutubro.pdf Acessado em 02 de março de 2017.

12. Dupont, J.; Dias, L. C.; Documento de Área 2009, CAPES. http://www. capes.gov.br/images/stories/download/avaliacao/QUIMICA19jun10.pdf Acessado em 02 de março de 2017.

13. Paniago, E. B.; Quim. Nova 1997, 20, 23.

14. CAPES, Orçamento - Evolução em Reais. http://www.capes.gov.br/ orcamento-evolucao-em-reais Acessado em 15 de março de 2017.

15. Santos, N. C. F.; Cândido, L. F. O.; Kuppens, C. L.; Quim. Nova, 2010, $33,489$.

16. https://pt.actualitix.com/search.php?q=PIB Acessado em 29 de março de 2017.

17. De Pierro, B.; Revista FAPESP, 2014, 8, 32. 